PALABRAS CLAVE

CEPAL

Publicaciones periódicas

Historia económica

Desarrollo económico

Desarrollo social

Desarrollo sostenible

Economistas

Sociólogos

Modelos de desarrollo

América Latina

Caribe

André Hofman

Director,

Revista de la CEPAL

œ andre.hofman@cepal.org

Miguel Torres

Editor Técnico,

Revista de la CEPAL

œ miguel.torres@ cepal.org
REVISTA DE LA CEPAL 96 - DICIEMBRE 2008

\section{El pensamiento cepalino en la Revista de la CEPAL (1976-2008)}

\author{
André Hofman y Miguel Torres
}

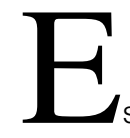

ste artículo examina el papel que ha desempeñado la Revista de Ia CEPAL como difusora del pensamiento cepalino y de otras corrientes analíticas que exploran los problemas del desarrollo. Para ello estudia una parte de la extensa colección de artículos que la revista publicó entre 1976 y el 2008, centrándose en aquellos que se aproximan de forma más nítida a las preocupaciones permanentes de la CEPAL (crecimiento y progreso técnico, pobreza e inequidad social, desarrollo sustentable y democracia y ciudadanía) y agrupándolos según el equipo directivo de la revista bajo el cual fueron publicados: Prebisch-Gurrieri, Pinto-Lahera y Altimir-Bajraj. Concluye con la presentación y somero análisis de los ensayos de Prebisch (1980), Pinto (1976) y Altimir (1994), alguna vez publicados en la Revista de la CEPAL. 


\section{I}

\section{Introducción}

En sus sesenta años de existencia, la CEPAL ha logrado difundir su pensamiento y el de sus intelectuales más connotados a través de un gran número de publicaciones periódicas o extraordinarias. Ejemplo de las primeras es el Estudio económico de América Latina y el Caribe, la publicación anual de más larga data de la CEPAL, nacida prácticamente con la fundación de nuestra casa y que ya cuenta con sesenta números a su haber. ${ }^{1}$ Cómo no mencionar, por ejemplo, la versión de 1948, donde se presenta el panorama global de la economía regional en perspectiva de largo plazo, o la de 1949, donde ya bajo el mandato de Prebisch se presentan sus visiones y las de la CEPAL acerca del progreso técnico, la relación de precios del intercambio y, en general, los factores determinantes del lento desarrollo en la región. ${ }^{2}$ No obstante lo anterior, dada la tendencia creciente del Estudio económico a especializarse en la macroeconomía de coyuntura y de largo plazo, ${ }^{3}$ aparecieron otras publicaciones periódicas de la CEPAL encargadas de dar cuenta de las situaciones y perspectivas en otros aspectos igualmente esenciales del desarrollo económico y social de la región, entre ellas el Panorama social de América Latina, el Panorama de la inserción internacional de América Latina y el Caribe, La inversión extranjera en América Latina y el Caribe y el Anuario estadístico de América Latina y el Caribe.

Sin embargo, más allá de esta variada gama de informes institucionales sobre los distintos aspectos del desenvolvimiento regional, gran parte de ellos orientados a la coyuntura, durante los últimos treinta y dos años la Comisión ha podido difundir una vasta producción de ideas y mensajes de carácter más académico y con particular énfasis en los aspectos estructurales del desarrollo, la que se ha plasmado en los artículos incluidos en la Revista de la CEPAL. Surgidos del seno mismo de la institución en algunos casos, o dando espacio a autores independientes o de otros organismos de dentro

\footnotetext{
${ }^{1}$ Véase un examen más detallado de la trayectoria de dicha publicación en CEPAL (2008a, cap. V).

${ }^{2}$ Véase CEPAL (1949) y CEPAL (1951), respectivamente.

${ }^{3}$ Los informes de coyuntura que entrega a mitad de año el Estudio económico son complementados en diciembre de cada año por los
}

o fuera de la región, en otros, los artículos de la revista han explorado, gracias a una independencia editorial rigurosa, un amplio espectro de temas, realidades regionales y nacionales y enfoques teóricos y metodológicos, tan rico como la vasta diversidad latinoamericana y caribeña.

A la luz del enorme acervo intelectual acumulado por nuestra revista, el presente artículo pretende recorrer los hitos que han marcado su existencia y reseñar sus principales contribuciones al pensamiento de la CEPAL. Para ello, en sus secciones II, III y IV se realiza un examen extenso, que por cierto no es exhaustivo, de aquellos artículos más representativos del pensamiento de nuestra casa, con matices y referencias ocasionales a autores - ya sea independientes o de otras instituciones- que indagan en los problemas del desarrollo desde posiciones no necesariamente similares ni del todo coincidentes con las cepalinas.

Las tres secciones señaladas abarcan tres etapas bien diferenciadas en la historia de la Revista de la CEPAL, marcadas respectivamente por cada uno de los tres binomios directivos que la han conducido desde su fundación. Así, la sección II examina los artículos más destacados que se publicaron bajo la conducción de Raúl Prebisch y su editor Adolfo Gurrieri; la sección III hace lo propio con respecto a la era de Aníbal Pinto Santa Cruz en la dirección y Eugenio Lahera en la edición técnica, y la sección IV considera contribuciones más recientes, publicadas bajo la dirección de Oscar Altimir y la subdirección de Reynaldo Bajraj.

Finalmente, en la sección V se hace un recorrido crítico de tres ensayos alguna vez publicados por la Revista de la CEPAL y escritos respectivamente por quienes en distintas etapas fueron sus Directores: Prebisch, Pinto y Altimir. Dichos artículos se reproducen in extenso en este número de la revista, a continuación del presente artículo. 


\section{II}

\section{La conducción Prebisch-Gurrieri (1976-1986)}

La Revista de la CEPAL fue inaugurada en 1976. Con una periodicidad inicial de dos números por año. El primer número fue publicado en el primer semestre de aquel año. ${ }^{4}$ Esta publicación vino a sustituir al Boletín Económico de América Latina, que apareció desde 1956 hasta la primera mitad de los años 1970.5

Según sintetiza Bielschowsky (1998), la CEPAL del decenio de 1970 está abocada temáticamente al tema de los llamados estilos de desarrollo, en un contexto internacional caracterizado por la dependencia, el peligroso y excesivo endeudamiento y la insuficiente capacidad exportadora de la región. Los análisis de esos años se plasman a través de ideas centradas en las estrategias de crecimiento, sus vínculos con la estructura productiva, los patrones distributivos y las estructuras de poder. Asimismo, hacen gran hincapié en la necesidad de las economías regionales de avanzar hacia un esquema de industrialización que compatibilice el mercado interno con el esfuerzo exportador. En el plano de las implicaciones de política, los mensajes principales son "viabilizar el estilo que lleve a la homogeneidad social" y "fortalecer las exportaciones industriales" (Bielschowsky, 1998, p. 23).

En suma, este decenio es testigo de la profundización de las ideas cepalinas de mediados del decenio de 1960 orientadas a incorporar con mayor fuerza la dimensión social del desarrollo y su estrecha vinculación con los aspectos económicos, en las cuales los problemas de la pobreza y la distribución de los ingresos empiezan a cobrar cada vez mayor relevancia. A estos esfuerzos también comienzan a incorporarse las primeras visiones de la CEPAL sobre desarrollo y medio ambiente.

Es en el contexto de estas ideas fuerza, entonces, que comienza el período fundacional de la Revista de la CEPAL. Su primer Director fue Raúl Prebisch, quien contó con el invaluable apoyo, como Secretario

\footnotetext{
${ }^{4}$ Posteriormente, a partir de 1979, la Revista de la CEPAL se transformó en una publicación cuatrimestral y hasta hoy se publica con regularidad en abril, agosto y diciembre de cada año.

${ }^{5}$ El Boletín era una publicación semestral. Ofrecía una reseña de la coyuntura latinoamericana que complementaba y actualizaba la que recogían los estudios económicos anuales de la Comisión. Además, publicaba artículos especiales sobre distintos temas relacionados con la economía regional, de carácter más estructural, así como notas informativas y metodológicas (véase CEPAL, 1974, página legal).
}

Técnico, del sociólogo argentino Adolfo Gurrieri. En su recordado artículo Cinco etapas de mi pensamiento sobre el desarrollo, Prebisch (1983) decía de la quinta de esas etapas que ella "se inició en realidad cuando, tras muchos años de fructífero servicio internacional, pude liberarme de las responsabilidades ejecutivas y la CEPAL me puso a cargo de su revista, donde resumí mis ideas en una serie de artículos que me sirvieron de base para escribir Capitalismo periférico. Crisis y transformación... Esta constituyó la quinta etapa, probablemente la última, de mi pensamiento sobre los problemas del desarrollo económico".

Raúl Prebisch ejerció la dirección de la revista desde 1976 hasta sus últimos días de vida. Su deceso se produjo en abril de 1986. En esos diez años de conducción, la revista no publicó solo las ideas finales de Prebisch sobre la naturaleza del capitalismo periférico o sus múltiples artículos sobre los estilos de desarrollo que dominaron el debate cepalino de la segunda mitad del decenio de 1970. Al respecto, cabe recordar ante todo que en la primera mitad del decenio de 1980 la región resintió los efectos de la crisis de la deuda, aquella que detonó "la década perdida" de América Latina. En ese contexto, las prioridades de la CEPAL se reorientaron más bien a lo coyuntural, y por ende el ajuste y sus shocks; la recuperación del crecimiento y el costo social de la estabilización macroeconómica se transformaron en los principales focos analíticos y de política para la institución.

La Revista de la CEPAL no estuvo ajena a estos debates. Publicó una gran cantidad de estudios relacionados con la crisis financiera del decenio de 1980, e incluso algunos anteriores a la crisis que intentaban advertir de ella. En un importante trabajo, Devlin (1979) señala los puntos de convergencia y divergencia entre los objetivos de la banca comercial y aquellos de los países en desarrollo. En un contexto regional de endeudamiento barato, Devlin pone una nota de escepticismo respecto de los incentivos de las instituciones prestamistas y los países acreedores, cuestionando la alineación de los intereses entre ambos grupos de agentes, y poniendo de relieve implícitamente los riesgos de insolvencia que esto podría generar en la región.

A partir de ese artículo, y una vez desatada la crisis, los análisis y perspectivas no dejaron de estar presentes 
en las páginas de la publicación. Destacan al respecto los artículos de Iglesias (1983) y Massad (1983). En el primero de ellos, el entonces Secretario Ejecutivo de la CEPAL planteaba que "en 1982 América Latina sufrió su crisis económica más profunda de todo el período de posguerra y, probablemente, la más grave desde los aciagos años de la Gran Depresión”. Mediante un análisis de variables macroeconómicas clave (crecimiento, desocupación, inflación y desequilibrios del sector externo), añadía que lo sucedido aquel año "en las economías de América Latina...resulta particularmente útil para comprender la naturaleza de la grave crisis económica que afecta a la región y sus causas, lo que constituye a su vez un conocimiento imprescindible para proponer medidas que la enfrenten con éxito".

Siguiendo una línea similar a la de Devlin (1979), el artículo de Massad (1983) examina el costo real del servicio de la deuda externa, demostrando la existencia de una diferencia de costos entre los acreedores y los deudores; propone un método alternativo para medir el costo real del servicio de la deuda y además esboza un análisis de los factores que lo determinan.

Otro tema importante de discusión durante estos complejos años fueron las primeras rondas de negociación orientadas a la reprogramación de la deuda. Al respecto, Devlin (1985) analizaba los resultados de estas rondas, anunciando "el ocaso de la gestión ortodoxa". El punto de partida de ese trabajo era el reconocimiento del deterioro de las condiciones de endeudamiento que la región experimentó durante las primeras rondas de negociaciones (1982-1983) e intentaba explicar, bajo la noción de monopolio bilateral, la posterior recuperación de ellas en la ronda 1984-1985.

Pero más allá de los numerosos artículos que la revista publicó sobre la crisis de la deuda, en esta primera etapa la publicación - al igual que en las dos siguientes- siempre mantuvo su preocupación por difundir las ideas centradas en los debates sobre el desarrollo de largo plazo, muchas de ellas relacionadas también con el pensamiento de la CEPAL.

Sobre la difusión del pensamiento cepalino a través de la revista, cabe mencionar que durante esta primera etapa, en 1978, la CEPAL cumplió sus primeros treinta años de existencia. Anticipándose a este acontecimiento, la revista publicó un año antes un artículo que daba cuenta de la evolución de las ideas de la CEPAL y sus vínculos con otras corrientes de pensamiento: el clásico texto de Cardoso (1977) denominado La originalidad de la copia: la CEPAL y la idea de desarrollo.

En esta obra, Fernando Henrique Cardoso examina en primer lugar las ideas fundacionales de Prebisch y la CEPAL sobre el desarrollo "y por qué generaron tanto alboroto" (Cardoso, 1977, p. 12), reseñando los principales aspectos de la noción prebischiana del sistema centro-periferia. Luego, "las vincula con otras posiciones doctrinarias y académicas que también alcanzaron cierta resonancia en la región...". El artículo analiza asimismo la forma en que estas ideas "modelaron políticas de desarrollo y se adaptaron a situaciones nuevas". El texto además relaciona el pensamiento cepalino con nuevas corrientes surgidas en los años 1960 y 1970 "referidas al estilo de desarrollo 'maligno', la dependencia estructural y el 'otro desarrollo" " (Cardoso, 1977, p. 7). La conclusión de Cardoso en esta obra es que más allá de las transformaciones que experimentó el pensamiento de la casa -al considerar los nuevos cambios globales, conocer otros aportes teóricos y nutrirse de ellos- la CEPAL tuvo la capacidad de preservar y demostrar la vigencia de su núcleo esencial de pensamiento: los factores estructurales del subdesarrollo, la importancia de incrementar la productividad para elevar la tasa de crecimiento de largo plazo a través del progreso técnico y la posibilidad de generar, de esta forma, mayores y mejores condiciones de bienestar en la periferia latinoamericana.

En relación con temas específicos del debate sobre el desarrollo de largo plazo, la revista constituyó una verdadera incubadora de ideas en las que connotados intelectuales y economistas de la región publicaron artículos que serían el inicio de obras importantes e ideas fuerza de gran resonancia en el debate académico y político. Según Torres Olivos (2006), estos son los años en que, por ejemplo, Fernando Fajnzylber está dedicado al análisis de las experiencias de crecimiento de largo plazo y las estrategias de industrialización de economías extrarregionales, tratando de establecer un paralelo con los procesos de América Latina. Fue precisamente en las páginas de la Revista de la CEPAL $\mathrm{N}^{\mathrm{o}} 15$ donde reflexionó acerca de la industrialización exportadora del sudeste asiático (Fajnzylber, 1981). Este artículo, junto a otros que analizaban la situación de las economías capitalistas avanzadas, constituyeron importantes insumos para dos de los conceptos más relevantes del autor: la "industrialización trunca" y el “casillero vacío" (Fajnzylber, 1983 y 1990).

En esta época también Celso Furtado se ocupaba de establecer las dimensiones culturales del desarrollo. Su planteamiento inicial concebía la cultura como un conjunto de partes cuyas interacciones guardan un cierto grado de coherencia. Más allá de esta noción, sostenía que la cultura es un sistema dinámico y por lo tanto afecto a cambios continuos, los que a su vez alteran el orden social en todas sus dimensiones, incluida la 
económica. El desarrollo debe concebirse entonces como un enriquecimiento del sistema cultural. En otras palabras, desarrollo y cultura están mutuamente supeditados. En efecto, el desarrollo consiste en potenciar las capacidades creativas del hombre, generando innovaciones culturales. No obstante lo anterior, Furtado distingue dos procesos de creatividad. De uno derivan innovaciones en el ámbito de lo que él denomina "cultura material", representada por el progreso técnico y la acumulación. De otro, derivan innovaciones que se dan en el ámbito de la cultura "no material", la cual está referida al conjunto de ideas y valores que una sociedad va construyendo. El desarrollo por la vía de la "cultura material" se logra a través de los excedentes económicos adicionales que amplían las opciones para los miembros de la comunidad. Las ideas sobre cultura material relativas a acumulación e innovación, especialmente en cuanto al rol del excedente, contenidas en su libro de 1978 titulado Criatividade e dependencia na civilização industrial, fueron sintetizadas también en el número 6 de la Revista de la CEPAL (Furtado, 1978).

En esta primera fase de la revista tampoco estuvieron ausentes las contribuciones cepalinas hechas desde la sociología del desarrollo. En momentos agitados y complejos en lo que a las condiciones políticas de la región se refiere (especialmente en el Cono Sur y particularmente en Chile), la CEPAL y su revista abordaron la contingencia política internacional, y desde este contexto, marcado por la guerra fría, analizaron en profundidad el vínculo entre desarrollo y democracia. Según Rodríguez (2006), las preocupaciones sobre el tema estuvieron presentes en las reflexiones de Prebisch sobre capitalismo periférico, que hacían hincapié en las nuevas bases para la consolidación democrática en las sociedades de la periferia; en las elaboraciones de Cardoso relacionadas con la reivindicación democrática y los movimientos sociales, y muy especialmente en Medina Echavarría y su visión renovadora de la democracia y sus contenidos. Respecto de las reflexiones de este último autor, dos artículos suyos plasmaron tales ideas en la Revista de la CEPAL. En el primero de ellos el sociólogo español proyectaba los distintos escenarios políticos por donde podría conducirse la región ante una inminente distensión entre las dos grandes potencias hegemónicas de aquel entonces (Medina Echavarría, 1976) y en el segundo indagaba en el futuro de las democracias occidentales, especialmente en América Latina (Medina Echavarría, 1977).

Como decíamos al comenzar la sección, esta fase de la revista estuvo marcada por el debate en torno a los estilos de desarrollo, desde el enfoque tanto económico como sociológico. El enfoque económico es tratado con más detalle en la sección V de este artículo. Por ahora, nos centraremos en el enfoque sociológico. Bielschowsky (1998) señala el papel que le cupo al número inaugural de la Revista de la CEPAL en la difusión de ideas en torno a los estilos de desarrollo. En efecto, de ese primer número destacan los artículos de Graciarena (1976) y Wolfe (1976). En el primero de ellos se hace un análisis crítico de las diversas acepciones de estilos de desarrollo, poniendo un mayor énfasis en aquellas nociones orientadas hacia los enfoques unificadores del desarrollo. Se rescata también el aporte desde lo social al enriquecimiento del concepto de estilo de desarrollo, considerando aspectos como la educación, la salud y la seguridad social, entre otros. El artículo de Wolfe, por su parte, aborda los diversos enfoques del desarrollo, examinando y cuestionando los objetivos y medios utilizados con frecuencia en el debate. Intenta el autor "sugerir una concepción existencial del desarrollo como un esfuerzo incesante para imponer una racionalidad valorativa dada a una realidad rebelde". Con este objetivo, el artículo finalmente "distingue los criterios principales que han sido usados para definir los fines y los medios del desarrollo (utópico-normativo, tecnocrático-racionalista y sociopolítico)", culminando con un análisis y crítica de la conducta de "los agentes de desarrollo".

Y si bien estos autores contribuyeron desde lo social hacia un concepto integral y multidisciplinario sobre el desarrollo y sus estilos, es importante considerar también los esfuerzos orientados a incorporar en el debate el medio ambiente y el desarrollo sustentable. La contribución a este debate desde la Revista de la CEPAL cristalizó en un artículo de Osvaldo Sunkel cuyo propósito era explorar los vínculos entre los estilos de desarrollo y el medio ambiente en América Latina (Sunkel, 1980). El artículo ofrece una descripción y una interpretación sistémica de los fenómenos que surgen a nivel regional en relación con el medio ambiente y el proceso de desarrollo. De este modo, en un marco conceptual amplio, Sunkel "analiza las transformaciones globales acaecidas en los últimos decenios, prestando especial atención a las consecuencias de diversa índole que la industrialización, la modernización agrícola y la urbanización han provocado sobre los factores ambientales y la manera en que éstos, a su vez, han repercutido sobre las posibilidades y límites del desarrollo" (Sunkel, 1980, p. 17).

El último número publicado bajo la dirección de Raúl Prebisch fue el 28, correspondiente a abril de 1986. Tras su fallecimiento, Adolfo Gurrieri continuó a cargo de la publicación como Secretario Técnico desde abril de aquel año hasta diciembre de 1987. Luego de 
ese período de transición un nuevo equipo asumió la conducción de la revista, poniendo fin así a una prolífica primera etapa en la que muchos de los autores clásicos del estructuralismo cepalino y latinoamericano contribuyeron a la producción y el debate de ideas a través de nuestras páginas.

\section{III}

\section{La conducción Pinto-Lahera (1987-1995)}

A partir del número 33 de la Revista de la CEPAL, publicado en diciembre de 1987, asumió la dirección de la revista el economista chileno Aníbal Pinto, quien estuvo acompañado durante todo su mandato por el cientista político chileno Eugenio Lahera en las labores de edición técnica. Luego de haber dirigido los once primeros números de la revista de economía política Pensamiento Iberoamericano, Pinto se hace cargo de la Revista de la CEPAL en la recta final del decenio de 1980. A nivel regional, el proceso político está signado por la recuperación del sistema democrático, especialmente en América del Sur. En el plano económico, los efectos de la crisis todavía afectan a los países latinoamericanos, especialmente en lo que respecta al costo social del ajuste derivado de las políticas de estabilización macroeconómica y de renegociación de la deuda (Bielschowsky, 1998). En el plano global, los eventos regionales se suceden en medio de la finalización de la guerra fría y el advenimiento de un nuevo orden internacional, dominado crecientemente por el neoliberalismo como doctrina y praxis de las reformas estructurales que tendrían lugar durante los años 1990 en todas las economías de la región.

En ese contexto regional e internacional, el debate interno de la CEPAL se centra en los procesos de ajuste y sus impactos sociales y en la reformulación de su propuesta para el desarrollo regional, la que intenta —en palabras de Rosenthal- construirse en una lógica de "continuidad y cambio" (Rosenthal, 1988).

A fines de la década de 1980, a través de dichos procesos y visiones renovadoras del desarrollo comienza a gestarse la principal idea-fuerza que dominaría el pensamiento y la acción de la CEPAL desde los años 1990 hasta la actualidad. Se trata de la propuesta de transformación productiva con equidad y del surgimiento del llamado enfoque neoestructuralista en el debate de los intelectuales cepalinos y latinoamericanos. También hay análisis de temas más específicos del desarrollo, como la pobreza y la distribución de los ingresos, la dimensión de género, el medio ambiente y el desarrollo sustentable. Todos ellos se suman a los aspectos más clásicos del pensamiento económico de la casa, vinculados al crecimiento de largo plazo, pero con esfuerzos crecientes por focalizar más los análisis en aspectos microsectoriales.

Todos los temas señalados, y muchos de los intelectuales que los desarrollaron, dejaron registro de sus elaboraciones en la Revista de la CEPAL. Sobre los temas macroeconómicos, especialmente aquellos relacionados con los efectos de la crisis y las perspectivas futuras, cabe mencionar en primer lugar el trabajo de González (1988), quien aborda la política macroeconómica para el desarrollo en el contexto del ajuste. Ahondando en los efectos y desafíos más específicos de la crisis de la deuda, Eyzaguirre (1989) analiza el comportamiento del ahorro y la inversión en un entorno de restricción externa y fiscal. Por otro lado, Mortimore (1989), con una perspectiva más microeconómica, estudia la conducta de la banca acreedora en la región mientras que, en ese mismo número de la revista, Devlin (1989) esboza a través de un enfoque más global las disyuntivas enfrentadas por la región como consecuencia del endeudamiento externo. Los planes de ajuste con crecimiento llevados adelante por las economías regionales para superar la crisis estuvieron doblemente condicionados por las principales instituciones financieras internacionales: el Fondo Monetario Internacional (FMI) y el Banco Mundial. En Meller (1989) se analiza esta doble condicionalidad y se plantea la necesidad de una coordinación mayor de los programas propiciados por uno y otro de estos organismos. Ramos (1989), en tanto, examina las nuevas corrientes académicas venidas desde "el Norte" en materia de teoría macroeconómica, haciendo hincapié en el debate entre "nuevos clásicos" y "neokeynesianos".

En lo que respecta a los análisis microsectoriales, se destacan los artículos publicados en este período en torno a los problemas del sector agroalimentario. Temas como los impactos del ajuste en el sector, las políticas sectoriales y la planificación macroeconómica, el análisis sectorial de la propia CEPAL, la seguridad alimentaria y las dimensiones sociales de la ruralidad constituyen 
aspectos profusamente abordados por diversos autores a través de nuestras páginas. ${ }^{6}$

Con el surgimiento de la propuesta de transformación productiva con equidad, basada en parte en los análisis previos de Fajnzylber (1983 y 1990), el sector industrial, considerado el principal portador de progreso técnico, recobró en el debate cepalino la importancia perdida tras las críticas neoliberales en los años más duros de la crisis de la deuda. No obstante lo anterior, la "nueva industrialización" planteada en dicha propuesta reconocía en primer lugar la necesidad de una apertura comercial competitiva y el desarrollo de complementariedades con los sectores primario y de servicios. No es de extrañar entonces que la Revista de la CEPAL haya dejado registro de estas ideas en artículos como el de Fajnzylber (1988), que analizaba la evolución y lecciones en materia de competitividad internacional y reestructuración productiva, así como la incorporación del progreso técnico, a través de un paralelo entre naciones industrializadas y en desarrollo. Tampoco dejan lugar a dudas, en esta temática de transformación productiva y cambio técnico, los trabajos de Lahera (1988) y Willmore (1989). En esta misma línea, pero referido al caso de Ecuador, cabe destacar el artículo de Hofman y Buitelaar (1994), que analiza las ventajas competitivas de este país y sus perspectivas de crecimiento en el largo plazo. Por su parte, en lo que respecta a las complementariedades sectoriales, Kuwayama (1989) aborda el potencial tecnológico del sector primario exportador. Se destacan también en este período los trabajos de Peres (1993 y 1994) y Rosales (1994), autores que tratan las políticas de competitividad y las políticas industriales. Otra idea-fuerza surgida a partir de la propuesta de transformación productiva con equidad es el concepto de "regionalismo abierto" como una alternativa de integración comercial, tema explorado por Fuentes (1994). Siempre en materia de integración, Rosenthal (1993) intenta responder, entre otras muchas, dos preguntas de gran relevancia: ¿En qué se diferencian los esquemas de integración de los países de la región de aquellos que se intentaron en los decenios de 1960 y 1970? ¿Cuáles son los instrumentos indicados para promover una integración intrarregional robusta?

Otros elementos clave de la propuesta fueron el cambio institucional y la valoración del sistema democrático como hábitat esencial para el desarrollo de un cambio productivo y social eficaz. En el primer caso, Fajnzylber (1991) aportó reflexiones en torno al papel

${ }^{6} \mathrm{Al}$ respecto, véase López Cordovez (1987), Harker (1987), Ortega (1988), Schejtman (1988) y Dirven (1993). del cambio institucional en la transformación productiva con equidad y, en un sentido similar, Lahera (1990) exploró la relación entre el Estado y dicha transformación. En el segundo caso, hubo un número importante de artículos que profundizaron en el papel que desempeña el sistema democrático en la transformación productiva con equidad. Vale mencionar entre ellos un trabajo de Enzo Faletto orientado a la exploración de los vínculos entre cultura y conciencia democrática, y otro que aborda las especificidades de los Estados latinoamericanos (Faletto, 1988 y 1989), así como un trabajo destacado de Graciarena (1988), sobre democracia y desarrollo, y otro de Wolfe (1990), sobre las estructuras sociales y el fortalecimiento democrático a las puertas del decenio de 1990. Un aspecto muy importante de la democracia, que en las décadas de 1990 y del 2000 adquiriría gran significación, es el de la ciudadanía. En un artículo señero sobre el tema, Calderón, Hopenhayn y Ottone (1994) sintetizan la propuesta cepalina de transformación productiva con equidad desde el ángulo cultural.

La propuesta de la CEPAL para los años 1990 considera la promoción de la equidad no solo como un imperativo ético del desarrollo, sino que también como una variable clave para el crecimiento, lo que difiere de las visiones neoclásicas que plantean la existencia de contraposiciones entre crecimiento y equidad. El surgimiento claro y potente del tema distributivo a través de la propuesta de transformación productiva con equidad, permitió un más sólido fortalecimiento de lo social en la visión cepalina del desarrollo. En esta plataforma más amplia de pensamiento adquirieron mayor preponderancia no solo las cuestiones asociadas a la distribución de los ingresos, sino también el enfoque integral de la pobreza. Además, desde la perspectiva de estos dos temas, estrechamente vinculados en la historia socioeconómica de la región, emergieron nuevos temas más específicos y profundos que han enriquecido durante los últimos veinte años las ideas y propuestas de la CEPAL. Hablamos, entre otras cosas, de la preocupación por las políticas sociales, las precariedades y disparidades en los mercados laborales de la región, la dimensión de género (fuertemente vinculada a la inequidad y la precariedad en el mundo del trabajo) y la juventud como sujeto de política social.

En esta su segunda etapa $-\mathrm{y}$, como veremos, aún más en la tercera- la Revista de la CEPAL publicó una gran cantidad de artículos sobre los temas de esta índole. Entre los trabajos orientados a presentar y medir las magnitudes de la inequidad y la pobreza como factores estructurales del subdesarrollo regional, destacan los de Altimir (1990 y 1994), Feres y León (1990) y 
Wolfe (1991). Entre aquellos referidos a las dimensiones y criterios generales de las políticas sociales sobresalen los de Durston (1988), Franco (1989), Sojo (1990), Rodríguez Noboa (1991), Cohen y Franco (1992) y Hopenhayn (1992). Entre los estudios sobre género, juventud y etnias destacan algunos como los de Krawczyk (1990 y 1993), López y Pollack (1989), Arriagada (1990 y 1994), Almeras (1994) y Durston (1992 y 1993). Finalmente, entre los artículos sobre trabajo y mercado laboral cabe mencionar los de Tokman (1988), Guerguil (1988), Infante y Klein (1991), Calderón (1993) y Rosenbluth (1994); asimismo, se publicó un trabajo pionero por el tema abordado y la significación que este adquiriría en el presente decenio: nos referimos a la protección social en América Latina y al trabajo de Uthoff (1995) sobre las reformas a los sistemas de pensiones en la región.

Tal como lo señala Torres Olivos (2006), una extensión importante de la propuesta de transformación productiva con equidad es su vínculo con el medio ambiente, los recursos naturales y el desarrollo sustentable en general, temas que por cierto ya habían sido introducidos en investigaciones anteriores de Sunkel y otros funcionarios de la CEPAL en los años 1970. Respecto al medio ambiente y el desarrollo sostenible cabe mencionar el análisis sobre desastres naturales y sus impactos socioeconómicos que efectúa Jovel (1989); los esbozos de Bustamante y Torres (1990) para una política ambiental eficaz; el enfoque de cuentas ambientales de Gligo (1990); las opciones de política orientadas a abatir la contaminación urbana (Durán de la Fuente, 1991); el trabajo de Valenzuela (1991) y su sugerente título - El que contamina, paga - relacionado con la aplicación de impuestos pigouvianos como instrumento de política ambiental; el artículo Participación y medio ambiente (Tomic, 1992) y el trabajo de Gligo (1995) sobre la situación y perspectivas del desarrollo sostenible en la región. Respecto de los problemas de los recursos naturales en la región se destaca el artículo de Dourojeanni (1994) sobre recursos hídricos y los trabajos de Sánchez Albavera (1993 y 1995), el primero de los cuales pone en el debate la situación de los recursos naturales en la región a inicios de los años 1990 y el segundo aborda el vínculo entre la globalización y la reestructuración energética en América Latina.

Finalmente, es preciso hacer mención de un debate generado entre fines del decenio de 1980 y comienzos del de 1990, que da pie al surgimiento del llamado neoestructuralismo. El debate asociado a este concepto se basa en los aportes de Fernando Fajnzylber y la propuesta cepalina de transformación productiva con equidad. Las ideas contenidas en esta propuesta - en donde se rescata el rol del progreso técnico, se propone una nueva industrialización que reconoce encadenamientos e interacciones con otras actividades productivas y se pone al centro de la propuesta tanto la equidad como la búsqueda de una inserción internacional auténticamente competitiva- incentivan a varios intelectuales y estudiosos del pensamiento de la CEPAL a integrar las ideas clásicas y las nuevas en un marco analítico que es denominado neoestructuralista. En este marco se agregan los temas emergentes que han acompañado el pensar y el actuar de la institución durante los últimos veinte años. Así, en el llamado neoestructuralismo cepalino se plasman más nítidamente las reflexiones sobre el medio ambiente y el desarrollo sustentable, la inequidad y la pobreza con análisis más focalizados, la integración comercial y la competitividad y el desarrollo productivo.

En torno al neoestructuralismo, la Revista de la CEPAL publicó una gran cantidad de artículos. En primer lugar cabe mencionar el trabajo de FfrenchDavis (1988), en el cual se ofrece un contrapunto entre la propuesta neoestructuralista y la doctrina neoliberal, tanto en sus dimensiones teóricas como en su aplicación al diseño de políticas públicas. En esta misma lógica de paralelos, Sunkel (1989) entrega una comparación entre el neoestructuralismo y el institucionalismo, con el propósito de explorar enriquecimientos mutuos entre ambas corrientes de pensamiento. Por último, Sunkel y Zuleta (1990) realizan otra comparación entre el neoliberalismo y el enfoque neoestructuralista, pero esta vez apuntan a los desafíos venideros de los años 1990 e indagan si las políticas recomendadas por una u otra doctrina contribuirían o no a que se retome la senda de crecimiento y desarrollo en la región.

En esta etapa de conducción de la revista, su Director Aníbal Pinto recibió en vida honores y reconocimientos por su enorme contribución intelectual a lo largo de una dilatada trayectoria profesional. Entre ellos sobresalen sus Doctorados Honoris Causa en la Universidad de Campinas (Brasil, 1989) y en la Universidad Nacional Autónoma de México (1991) y el Premio Nacional de Humanidades y Ciencias Sociales que le otorgó el Gobierno de Chile en septiembre de 1995, meses antes de su deceso. Junto a todos estos reconocimientos, corresponde hacer hincapié en la gran contribución de Pinto en la dirección de la Revista de la CEPAL, donde impulsó de manera protagónica el proceso de renovación del pensamiento cepalino en el cual él también tuvo gran influencia. 


\section{IV}

\section{La conducción Altimir-Bajraj (1996-agosto del 2008)}

Tras el fallecimiento de Pinto, y luego de la publicación del número 58 en abril de 1996, que estuvo a cargo del Secretario Ejecutivo de la CEPAL Gert Rosenthal y de Eugenio Lahera como Secretario Técnico, asumió la dirección de la revista el economista argentino Oscar Altimir, quien se desempeñó en el cargo hasta agosto del 2008. En este período la publicación exhibió logros importantes, tanto en términos de una mayor difusión como de más acercamiento al mundo académico extracepalino y de ampliación de su línea editorial. Desde el 2003 la labor de Altimir se vio también beneficiada por la colaboración del economista argentino Reynaldo Bajraj como Director Adjunto de la revista. Tanto Altimir como Bajraj desarrollaron una fructífera carrera funcionaria en labores sustantivas de la CEPAL, la que llevó a cada uno de ellos a ocupar, en su momento, el cargo de Secretario Ejecutivo Adjunto de la Comisión. Toda esa experiencia alcanzada contribuyó significativamente a los logros de la Revista de la CEPAL en los últimos años. ${ }^{7}$

Muchos de los artículos publicados en esta etapa incorporaron herramientas analíticas que se hallan en la frontera del conocimiento (análisis econométricos, modelos de equilibrio general computables, análisis sectoriales más refinados y otras), lo que ha permitido que, desde diciembre del 2007, nuestra publicación esté incorporada en el Social Sciences Citation Index (SSCI) que publica Thomson ISI.

Estos importantes avances se han traducido en mejoramientos de la calidad del material publicado y el reforzamiento de la línea editorial de la revista, basada en una independencia académica e intelectual irrestricta. En cuanto a los asuntos abordados en la publicación,

\footnotetext{
${ }^{7}$ Oscar Altimir estuvo vinculado a la CEPAL desde mediados de los años 1960 y ocupó altos cargos en ella: Director de la División de Estadística y Análisis Cuantitativo (1976-1983), de la División Conjunta CEPAL/ONUDI de Industria y Tecnología (1984-1988) y de la División de Desarrollo Económico (1989-1993); entre 1994 y 1996 fue Secretario Ejecutivo Adjunto de la Comisión. Reynaldo Bajraj se vinculó a la CEPAL en 1976, desempeñándose en diversos cargos del ILPES: Experto en Política Económica, Director del Programa de Investigaciones y Director del Programa de Asesorías. En 1987 fue nombrado Director del Centro Latinoamericano de Demografía de las Naciones Unidas (CELADE) y entre 1997 y el 2003 se desempeñó como Secretario Ejecutivo Adjunto de la CEPAL.
}

se ha mantenido un adecuado equilibrio entre los temas económicos, la visión del desarrollo y el largo plazo y los temas sociopolíticos.

Antes de referirnos a los trabajos más destacados en torno a estas líneas recurrentes de investigación, nos parece necesario destacar cuatro hitos importantes en la trayectoria de la Revista de la CEPAL: la publicación en octubre de 1998 del Número Extraordinario con motivo del cincuentenario de la casa, la conmemoración del centenario del natalicio de Raúl Prebisch en el número 75, la publicación a partir del 2002 de las ponencias que han presentado connotados intelectuales en las versiones anuales de la Cátedra Raúl Prebisch y, en el 2005, una edición extraordinaria de artículos de la Revista de la CEPAL publicados entre 1995 y 2004, traducidos al francés.

Como ya se dijo, la CEPAL conmemoró su quincuagésimo aniversario en 1998. Con motivo de esta celebración, la revista publicó un Número Extraordinario en el mes de octubre, constituido por una treintena de artículos de los más prestigiosos profesionales relacionados - interna o externamente- con la trayectoria de la acción y el pensamiento de la CEPAL. Un artículo importante de este número es el trabajo de Bielschowsky (1998), material recurrente como referencia sobre la evolución de ese pensamiento. Por su parte, Katz (1998) aborda las lecciones y desafíos del aprendizaje técnico, en el contexto de un área clásica de las ideas cepalinas: el desarrollo industrial de la región. Por otro lado, la importancia que la Comisión dio al fenómeno de la globalización desde fines de los años 1990 — es decir, a partir del período de José Antonio Ocampo en la Secretaría Ejecutiva - tuvo su expresión en este Número Extraordinario a través de los artículos La globalización y la gobernabilidad de los países en desarrollo de Bouzas y Ffrench-Davis (1998), América Latina y la globalización de Aldo Ferrer (1998) y un estudio de Di Filippo (1998) que mira la noción centro-periferia a la luz de los años 1990. Destacan también en este número el ensayo de Assael (1998) en torno al desafío de la equidad en la región, y dos estudios sobre los obstáculos a la integración regional. El primero es de Sunkel (1998) y plantea un interrogante: ¿es la integración funcional a 
los objetivos del desarrollo? El segundo es de Urquidi (1998) y aborda desde el punto de vista histórico "los incidentes de integración" en Centroamérica y Panamá durante los años 1950.

El 2001 fue un año significativo para la CEPAL y su revista, por cuanto correspondió al centenario del natalicio de Raúl Prebisch. Se consideró oportuno no solo homenajearlo sino también resaltar sus aportes al pensamiento sobre el desarrollo. Por lo tanto, el número 75 de la Revista dedicó una sección de más de cien páginas a material sobre su obra. Abre este homenaje una entrevista hasta entonces inédita a Prebisch realizada por Pollock, Kerner y Love (2001) y lo continúa un ensayo en el que Ocampo (2001) vincula algunas ideas relevantes del pensamiento prebischiano con la agenda de desarrollo de América Latina para el nuevo siglo. El homenaje también incluye los trabajos de Rodríguez (2001), O'Connell (2001), Gurrieri (2001), quien se encarga de reseñar las ideas del joven Prebisch, y Cortés Conde (2001) y su ensayo histórico sobre los años en que Prebisch se desempeña en distintos cargos como funcionario del gobierno argentino, especialmente en el Banco Central. La sección concluye con los trabajos de Dosman (2001) y González (2001), el primero sobre las relaciones Estado-mercado en la óptica y evolución del "manifiesto" de Prebisch y el segundo centrado en el proceso de industrialización de América Latina según la concepción de Prebisch y la CEPAL, en contraste con los procesos correspondientes de los Estados Unidos (según el enfoque de Alexander Hamilton), de Alemania (bajo el foco de Frederick List) y un caso más general bajo el prisma neoclásico de John Stuart Mill.

En agosto del 2001, en el marco de las conmemoraciones vinculadas al natalicio de Prebisch, la CEPAL inauguró la cátedra que lleva su nombre y cuyo primer expositor fue Celso Furtado. En el 2002 correspondió al profesor y Premio Nobel de Economía, Joseph Stiglitz dictar la segunda Cátedra Prebisch, que estuvo referida a la evolución e impactos de las reformas en América Latina. Desde ese momento la Revista de la CEPAL ha publicado ininterrumpidamente, en calidad de artículos, las cátedras dictadas hasta el año 2007. Así, es posible citar el ya mencionado artículo de Stiglitz (2003); el de Cardoso (2004), que analiza las interacciones entre política y desarrollo económico; el de Ricúpero (2004) y sus reflexiones en torno a la vigencia de las ideas prebischianas; el de Rodrik (2005), que aborda la diversificación económica; de Iglesias (2006), que explora el papel del Estado y los paradigmas económicos en América Latina, y el de Halperin (2008), que recoge los aspectos más destacados de su notable ponencia en torno al contexto histórico de la CEPAL, presentada en la Cátedra Prebisch del 2007.

En el 2005 la Revista de la CEPAL marcó un hito de gran importancia al editar un número especial con una compilación de artículos publicados entre 1995 y el 2004, pero ahora traducidos al francés. El hecho tuvo enorme significación, por cuanto la revista pudo mostrar al mundo académico y político francés - históricamente vinculado con el origen y la misión de la CEPAL — una amplia gama de trabajos de gran calidad que hasta esa fecha solo habían tenido llegada en los medios de habla inglesa e iberoamericanos. Este proyecto de la CEPAL contó con el respaldo de instituciones de cooperación y académicas de Francia, a través del Ministerio de Relaciones Exteriores de ese país y del Institut des hautes études pour l'Amérique Latine.

El número especial en francés contiene una colección de 10 de los artículos que se publicaron en la Revista de la CEPAL y en CEPAL Review durante el período ya indicado, y además dos ensayos de los académicos franceses David Dumoulin Kervran y Jean-François Deluchey. Uno trata de las políticas de conservación del medio ambiente en nuestra región en situaciones de internacionalización y de convergencia de estilos políticos (Kervran, 2005), y el otro analiza el pasado y las perspectivas de los esquemas de seguridad interior en los países de América Latina (Deluchey, 2005).

Naturalmente, los otros diez ensayos guardan relación con las principales líneas de investigación de la CEPAL y su revista, entre ellas la macroeconomía del desarrollo, el cambio técnico y el crecimiento de largo plazo, los aspectos sociodemográficos del desarrollo y otros diversos tópicos centrados en las finanzas públicas y la integración y el comercio.

Haremos mención enseguida a algunos de los artículos incluidos en el número en francés, pero refiriéndonos a sus versiones originales en español. Un artículo destacado de esta colección es el ensayo de Ocampo (1999), en el cual se argumenta que la agenda de reforma financiera internacional debiera ampliarse al menos en dos sentidos: debería ir más allá de la prevención y solución de las crisis y "debería tener en cuenta no solo el papel de las instituciones mundiales sino también el de las regionales y la definición explícita de las áreas en que convendría preservar la autonomía nacional".

Otro ensayo que resalta es el de Klein y Tokman (2000), cuyo objetivo es analizar los impactos de la globalización en el mercado del trabajo y en la estratificación social. Reconociendo el consenso existente acerca de los beneficios que generaría la globalización para las naciones del mundo, los autores manifiestan sus dudas 
sobre los beneficios potenciales netos de este fenómeno, especialmente en lo que respecta a la distribución de tales beneficios. Siempre en torno a la globalización, Frenkel (2003) analiza las crisis financieras y cambiarias que afectaron a América Latina bajo el contexto de la globalización del capital.

Por su parte, Escaith (2001) examina a la luz de la globalización y bajo el marco analítico de las teorías endógenas del crecimiento el caso de las economías de pequeña escala en América Latina y el Caribe. Desde la perspectiva del desarrollo productivo sectorial, Katz (2000) estudia los cambios estructurales y la productividad del sector industrial de la región durante el período 1970-1996. En una dirección similar, pero más genérica y transversal a los distintos sectores productivos, Pérez (2001) "propone una interpretación del desarrollo como proceso de acumulación de capacidades tecnológicas y sociales, en función del aprovechamiento de ventanas de oportunidad sucesivas y distintas", las que a su juicio estarían determinadas por las revoluciones tecnológicas provenientes de las economías avanzadas.

Una propuesta sugerente - surgida esta vez desde una combinación entre análisis macroeconómico y de los aspectos sociales del desarrollo- se expone en el artículo de Stallings y Weller (2001), que aborda la evolución de los mercados laborales latinoamericanos y caribeños durante la década de 1990 y recalca la importancia del empleo como cimiento principal de la política social en los países de la región.

La educación y las políticas de acumulación de capital humano están estrechamente ligadas al mundo laboral y social, por cuanto fortalecen el desarrollo de capacidades individuales y aumentan las oportunidades de las personas para acceder a más y mejores empleos, especialmente para los y las jóvenes (CEPAL, 2008b). En 1992, la propuesta de transformación productiva con equidad se hizo extensiva a múltiples áreas temáticas. Cuando aún se contaba con la orientación de Fernando Fajnzylber, se abordó precisamente la educación (y el conocimiento) como eje de esa transformación (CEPAL/ UNESCO, 1992). En ese trabajo tuvo una participación importante, además de muchos otros profesionales, el experto en educación Juan Carlos Tedesco. Diez años después de esa propuesta interinstitucional, Tedesco y López (2002) examinaron los desafíos enfrentados por la educación secundaria en América Latina, haciendo hincapié en la cobertura y calidad de este nivel educativo.

Aparte de estos artículos incorporados en el número especial en francés, en esta tercera etapa de la Revista de la CEPAL se publicaron otros interesantes trabajos relacionados con el pensamiento de la casa y las elaboraciones de otros cientistas sociales de la región. En el ámbito del pensamiento cepalino surge el ensayo de Ocampo y Parra (2003), que analiza la evolución de los términos de intercambio entre productos básicos y bienes manufacturados. Tomando como base la tesis seminal de Prebisch y Singer sobre el deterioro secular de la relación de precios del intercambio (idea que fue un elemento clave en el pensamiento de Prebisch y la CEPAL en el decenio de 1950) y haciendo uso del instrumental analítico de la econometría de series de tiempo, Ocampo y Parra determinan el comportamiento tendencial de 24 productos básicos, concluyendo que "las profundas transformaciones que enfrentó la economía mundial alrededor de 1920 y de 1980 se tradujeron en un deterioro escalonado, que se reflejó a largo plazo en una caída cercana al $1 \%$ anual de los índices agregados de los precios relativos de las materias primas".

En este período, los temas macroeconómicos han tenido una importante presencia en la revista, desde el punto de vista tanto fiscal como de los ciclos financieros y reales y el crecimiento de largo plazo. Mencionaremos solo algunos de estos trabajos, ya que citarlos a todos escapa a los alcances de este documento. En primer lugar cabe nombrar a Heymann (2000) quien analiza las relaciones entre los shocks macroeconómicos, las expectativas y las respuestas de política. Martner (2000), por su parte, examina el papel de los estabilizadores fiscales. Continúa Morley (2000) y su exploración sobre los efectos distributivos del crecimiento y las reformas estructurales en América Latina en el decenio de 1990. Moguillansky (2002) analiza la inversión y la volatilidad financiera en la región en el decenio de 1990; usando análisis econométricos, la autora concluye que si bien la afluencia de capital hacia los países de la región tuvo efectos positivos, estos se vieron atenuados por los efectos negativos de la volatilidad asociada a tales corrientes financieras. Por su parte, Ibarra (2004) indaga, con una perspectiva histórica, en la adopción de reformas importadas en el marco de los distintos "factores rectores del orden económico internacional". En esta perspectiva macro, los últimos análisis de la CEPAL sobre la sostenibilidad del crecimiento económico han apuntado a la necesidad de reducir la volatilidad real, lo que subraya la importancia del papel que puedan desempeñar las instituciones financieras regionales. Estos vínculos son los analizados precisamente en Machinea y Titelman (2007).

En los tres últimos años, los análisis sobre las políticas fiscales retornaron a las páginas de la revista. Así, Paunovic (2005) aborda la sostenibilidad de la 
deuda pública en la región; Jiménez y Tromben (2006) estudian el auge de los precios de los recursos naturales no renovables (entre el 2003 y el 2007), la bonanza generada en las finanzas públicas a partir de ese auge y sus implicaciones en materia de política fiscal; Ocampo (2007) se aboca a la macroeconomía de la bonanza económica y Aldunate y Martner (2006) examinan la protección social desde el ángulo de las políticas de Hacienda.

En lo que se refiere a los aspectos políticos, sociales y demográficos del desarrollo, Hopenhayn (2001) trata las formas tradicionales y emergentes de ciudadanía; Sojo (2001) analiza las reformas de gestión en salud en la región; Schkolnik y Chackiel (2004) relacionan los sectores más postergados de la región y la transición de la fecundidad; Saraví (2004) aborda la segregación urbana y el espacio público en la Argentina con posterioridad a la crisis del 2001, apuntando en especial a los segmentos juveniles de los enclaves de pobreza estructural. También desde una perspectiva demográfica, Dirven (2004) explora la dinámica del empleo rural no agrícola (ERNA) desde los años 1990 y los distintos factores que lo determinan, postulando "que la localización y las diversas 'distancias' que la acompañan constituyen un elemento central del ERNA". El ensayo de Rodríguez (2005), relativo a Chile y con aportes desde lo demográfico, planteó un tema de gran importancia que fue retomado en trabajos posteriores de la CEPAL: la reproducción en la adolescencia. Otro tema social, especialmente por sus implicaciones de política pública, es el que trata Villatoro (2005); este autor se refiere a los programas de transferencias monetarias condicionadas, sintetizando los casos de América Latina.

Lo laboral ha sido siempre objeto de estudio y análisis de política social. Tanto desde los enfoques de demanda como de oferta, en su calidad de factor productivo y fuente de crecimiento de largo plazo, así como por su naturaleza dual - al tratarse de la acción transformadora del hombre en el proceso de producción y del derecho de los individuos a esta vía de subsistencia y bienestar-, el empleo, el mercado laboral y especialmente sus vínculos con la protección social han sido temas permanentemente tratados en la Revista de la CEPAL.

En esta línea de investigación se halla el ensayo de Vergara (2005), que analiza la dinámica laboral de Chile a nivel de plantas industriales, con énfasis en los procesos de creación y destrucción de empleos. Se trata de un enfoque de demanda de trabajo que recurre a técnicas econométricas de panel, específicamente de métodos generalizados de momentos (MGM). En estos marcos teórico y metodológico, el autor encuentra evidencia de la naturaleza procíclica de la creación de empleos y de la naturaleza anticíclica de su destrucción. Asimismo, los resultados muestran que la liberalización comercial aumenta la rotación de empleo.

Con un enfoque más centrado en la oferta, Carlson (2002) analiza el vínculo entre los logros educativos y la posibilidad de obtener empleo (empleabilidad) e ingresos laborales en algunos países de la región. Recurriendo a la metodología de cálculo de la rentabilidad de la inversión en capital humano, diferenciando por nivel educativo y por sexo, la autora encuentra, como es de esperar, rentabilidades positivas para esa inversión y concluye que es necesario conducir las políticas públicas hacia la generación de una fuerza de trabajo más competitiva en virtud de una mayor y mejor dotación de capital humano, que esté en mejores condiciones de asumir la fuerte competencia impuesta por el proceso de globalización.

Una arista muy singular de las fallas en los mercados laborales de América Latina y el Caribe ha sido la compleja inserción de los jóvenes. Weller (2007) analiza precisamente las debilidades de la empleabilidad juvenil, argumentando que el desempleo de la juventud no solo afecta el bienestar de este segmento demográfico, sino también algunos factores clave del desarrollo de largo plazo.

Finalmente, dentro de los temas laborales, la Revista de la CEPAL logró en esta tercera etapa difundir los principales mensajes y propuestas de la Comisión en materia de protección social y sistemas de pensiones.

Entre ellos destacan dos trabajos de Mesa-Lago (1996 y 2004): el primero analiza la posición de los organismos internacionales y regionales frente al proceso de reformas a los sistemas de pensiones en varios países de América Latina durante los años 1990 y el segundo, desde una perspectiva temporal más amplia, evalúa las reformas estructurales de estos sistemas, comparando tres tipos de reformas aplicados en 12 países de la región. Jiménez y Cuadros (2003), por otro lado, analizan la cobertura de los sistemas de pensiones, planteando la necesidad de ampliarla. Dos trabajos basales de la propuesta de la CEPAL sobre protección social son los de Uthoff (2002 y 2006): el trabajo del 2002 aborda el esencial vínculo entre mercados laborales y sistemas de pensiones, en tanto que el del 2006 analiza las reformas a tales sistemas en relación con las brechas de bienestar. Titelman y Uthoff (2003) examinan el papel del aseguramiento para la protección social. Teniendo en cuenta que los sistemas y políticas de salud siempre han tenido vínculos con los sistemas de pensiones, Titelman (1999) estudia las reformas al financiamiento de la salud en 
Chile, describe "el modelo de financiamiento chileno y plantea la necesidad de redefinir la actual configuración público-privada en el sector salud, para permitir más solidaridad en el financiamiento, reducir el problema de la selección de riesgos y permitir una mejor articulación entre el subsector privado y el subsector público, tanto en la esfera de lo financiero como en el ámbito de la provisión de servicios de salud".
En síntesis, esta tercera etapa de la Revista de la CEPAL concluye con un amplio espectro de temas del desarrollo, en los que se ha ido profundizando desde las visiones más generalistas a las más específicas, enriqueciendo los análisis con enfoques e instrumentos analíticos más refinados y poniendo a la mayoría de los artículos publicados en las cercanías de la frontera del conocimiento y del rigor e independencia intelectuales.

\section{V \\ Las ideas de Prebisch, Pinto y Altimir plasmadas en la Revista de la CEPAL}

En esta sección final del trabajo nos referiremos a los tres ensayos que le seguirán, testimonio de los grandes hitos y personas que forjaron la historia de la Revista de la CEPAL. Con esos ensayos, publicados alguna vez en la revista y cuyos autores respectivos son Prebisch, Pinto y Altimir, se busca rescatar y poner en perspectiva los aportes teóricos de los Directores de la revista a sus páginas.

El primero de los ensayos indicados es el de Raúl Prebisch "Hacia una teoría de la transformación" (Prebisch, 1980), que puso término a una serie de artículos en los cuales dicho autor expuso sus últimas elaboraciones en torno al capitalismo periférico (Prebisch, 1976, 1978 y 1979). ${ }^{8}$ En el artículo de 1976 había presentado su visión y crítica del modelo capitalista imperante en las economías de la periferia, en el de 1978 se había referido a la estructura y crisis del sistema y en el de 1979 había reflexionado acerca de las visiones neoclásicas del liberalismo económico. El objetivo esencial de esos tres artículos, además de entregar un análisis crítico del modelo capitalista en la periferia, había sido sin duda el de demostrar teóricamente la incapacidad de la ortodoxia neoclásica para interpretar adecuadamente ese modelo. Partiendo de una reseña de estos aspectos, en "Hacia una teoría de la transformación" Prebisch esboza lineamientos de políticas tendientes a la transformación del sistema, en los que subyacen elementos que rescatan valores del liberalismo y el socialismo; pone al centro de la transformación la esencialidad de la democracia y, finalmente, aborda aspectos económicos de la trans-

\footnotetext{
${ }^{8}$ Estos estudios fueron la base de su último libro (Prebisch, 1981).
}

formación, recurriendo a conceptos que habían estado presentes en toda su obra desde los trabajos pioneros de fines del decenio de 1940: el progreso técnico, los patrones de demanda, la estructura productiva y las especificidades del capitalismo periférico.

En su momento, este ensayo de nuestro primer Director prometió y generó polémica; sin embargo, amerita una relectura profunda a la luz de la coyuntura crítica de este primer decenio del siglo XXI, cuando los fundamentos macroeconómicos ortodoxos se tornan más débiles frente a la posibilidad real de una crisis financiera mundial que se asemejaría, según muchos economistas, a la gran depresión de los años treinta. En el mar de incertidumbres por el que atraviesa la humanidad, da luces respecto de la necesidad de los pueblos latinoamericanos de transformar sus modelos de producción, consumo y relaciones societales para poder reducir la vulnerabilidad a las crisis cíclicas del capitalismo, por un lado, y para corregir, por el otro, las fallas estructurales que este modelo ha mostrado permanentemente en el mundo periférico: la desigualdad y la pobreza, la escasa diversificación productiva, un crecimiento lento y volátil y el agotamiento de las ricas reservas de biodiversidad que caracterizan a nuestro continente.

El ensayo de nuestro segundo Director, Aníbal Pinto, titulado "Notas sobre los estilos de desarrollo", aborda este tema surgido en el decenio de 1970 pero que mantiene plena validez en la actual fase de la globalización (Pinto, 1976). El trabajo apunta a los aspectos económicos que hacen a un estilo de desarrollo. Pinto precisa de partida el concepto de estilo, que básicamente se asocia al modo en que una sociedad se organiza a través de un determinado sistema económico para resolver tres 
cuestiones esenciales: qué, cómo y para quién producir. En la idea de estilo de desarrollo se conjugan entonces dos conjuntos de rasgos clave. En primer lugar están los factores de tipo estructural como i) la organización de la producción, ii) la estructura sectorial del producto y el empleo, iii) el progreso técnico incorporado y iv) el modelo de inserción internacional. Los factores estructurales i), ii) y iii) sin duda encuentran su base en una contribución conceptual previa del mismo Pinto y una de las más importantes en su obra, la llamada "heterogeneidad estructural" (Pinto, 1970). En segundo lugar se hallan los factores dinámicos que hacen a un estilo de desarrollo, los que tienen relación principalmente con las características propias de la demanda, es decir, su nivel, su composición y, como antecedente de ellos, la distribución del ingreso.

Por la notable vigencia de este ensayo su relectura es altamente recomendada, con los mismos argumentos con que se recomienda releer Prebisch (1980). Las respuestas que no dan las visiones ortodoxas de cualquier cuño, la profundización de las desigualdades sociales a escala global, la especialización, el deterioro ambiental y las amenazas del calentamiento global, son realidades objetivas que impulsan a replantearse las formas de producir y de organizar las sociedades a través de estilos de desarrollo inclusivos y sustentablemente prósperos. Revitalizar estos debates a través de los enfoques de Pinto y otros estudiosos de los estilos de desarrollo puede aportar antecedentes muy útiles para generar nuevas reflexiones en torno a temas apremiantes como este.

Nuestro tercer Director, Oscar Altimir, también ha publicado artículos en la Revista de la CEPAL, sobre todo antes de ocupar ese cargo. Él se ha especializado por más de treinta años en dos de los factores estructurales más característicos del subdesarrollo latinoamericano: la pobreza y la distribución de los ingresos. Sus aportes cuantitativos y analíticos constituyen un acervo invaluable que ha influido tanto en los especialistas cepalinos como en el mundo académico externo. Muchos de estos aportes se materializaron en publicaciones de la Comisión y de entidades académicas. Todos sus artículos incluidos en la Revista de la CEPAL han versado invariablemente sobre la pobreza y la distribución del ingreso en América Latina y el Caribe (Altimir, 1981, 1990, 1994 y 2002).

El ensayo de Oscar Altimir que comentamos aquí se titula Distribución del ingreso e incidencia de la pobreza a lo largo del ajuste. Ya el título indica que trata de los efectos de la crisis de los años 1980 y sus procesos de estabilización macroeconómica sobre la distribución del ingreso, y cómo estos procesos incidieron en la pobreza. En rigor, lo que se analiza es precisamente el costo distributivo de la crisis y el ajuste, por un lado, y por otro los efectos distributivos de la recuperación y la reanudación del crecimiento sostenido a principios de los años 1990. Mediante comparaciones cuantitativas de 10 países de América Latina, Altimir estudia los cambios en la distribución de los ingresos y en ciertos indicadores macroeconómicos importantes, como la tasa de inflación y la tasa de crecimiento. Además, compara la distribución del ingreso antes y después de ajuste.

El trabajo de Altimir tiene el mérito no solo de ser uno de los trabajos señeros de la amplia gama de obras producidas en la CEPAL sobre pobreza y distribución del ingreso, sino además el de haber tenido dos grandes aciertos. El primero fue anticipar a comienzos de los años 1990 un hecho que se ha podido corroborar con el correr del tiempo: aunque el crecimiento en el corto plazo y la reducción de la inflación tienen un efecto de mitigación de la pobreza, el crecimiento sin mejoras distributivas —es decir, sin haberse concretado la transformación productiva con equidad-implica en el mediano y el largo plazo un abatimiento más lento de la pobreza. El segundo acierto fue criticar las modalidades tradicionales y las nuevas formas de política pública, influidas ya plenamente por las reformas propugnadas en el Consenso de Washington, aventurando que ellas tenderían a elevar los niveles de desigualdad.

Estas conjeturas pesimistas se han materializado con creces, dando cuenta de un mundo marcado por enormes y crecientes asimetrías en la distribución de la riqueza e ingentes masas humanas que viven en condiciones de pobreza. Por lo mismo, el estudio de Altimir tiene un alto grado de vigencia, en especial ante la inminente recesión mundial. Tanto la pobreza, que era aún elevada incluso en años de auge regional (2003 a 2007), como la persistencia de la inequidad podrían empeorar por los efectos inmediatos de la crisis y por los costos distributivos que podrían derivar de los ajustes necesarios para la estabilización macroeconómica. El estudio invita a una reflexión profunda - especialmente desde el ámbito normativo- sobre el desarrollo de largo plazo y al diseño de políticas públicas que mitiguen los factores estructurales del subdesarrollo, con la misma determinación con que hoy se aplican múltiples políticas de estabilización de corto plazo con las cuales los gobiernos del mundo y los organismos financieros internacionales intentan el salvataje de la banca mundial y el capital especulativo. 
Aldunate, E. y R. Martner (2006): Política fiscal y protección social, Revista de la CEPAL, No 90, LC/G.2323-P, Santiago de Chile, diciembre.

Almeras, D. (1994): Logros y obstáculos en la educación formal de las mujeres, Revista de la CEPAL, N ${ }^{\circ}$ 54, LC/G.1845-P, Santiago de Chile, diciembre.

Altimir, O. (1981): La pobreza en América Latina: un examen de conceptos y datos, Revista de la CEPAL, No 13 , E/CEPAL/G.1145, Santiago de Chile, abril.

(1990): Desarrollo, crisis y equidad, Revista de la CEPAL, $\mathrm{N}^{\circ}$ 40, LC/G.1613-P, Santiago de Chile, abril.

(1994): Distribución del ingreso e incidencia de la pobreza a lo largo del ajuste, Revista de la CEPAL, N 52, LC/G.1824-P Santiago de Chile, abril.

Altimir, O., L.A. Beccaria y M. González Rosada (2002): La distribución del ingreso en Argentina, 1974-2000, Revista de la CEPAL, $\mathrm{N}^{\circ} 78$, LC/G.2187-P, Santiago de Chile, diciembre.

Arriagada, I. (1990): La participación desigual de la mujer en el mundo del trabajo, Revista de la CEPAL, No 40, LC/G.1613-P, Santiago de Chile, abril.

(1994): Transformaciones del trabajo femenino urbano, Revista de la CEPAL, No 53, LC/G.1832-P, Santiago de Chile, agosto.

Assael, H. (1998): La búsqueda de la equidad, Revista de la CEPAL, número extraordinario, LC/G.2037-P, Santiago de Chile, octubre.

Bielschowsky, R. (1998): Evolución de las ideas de la CEPAL, Revista de la CEPAL, número extraordinario, LC/G.2037-P, Santiago de Chile, octubre.

Bouzas, R. y R. Ffrench-Davis (1998): La globalización y la gobernabilidad de los países en desarrollo, Revista de la CEPAL, número extraordinario, LC/G.2037-P, Santiago de Chile, octubre.

Bustamante, M.I. y S. Torres (1990): Elementos para una política ambiental eficaz, Revista de la CEPAL, No 41, LC/G.1631-P, Santiago de Chile, agosto.

Calderón G., F. (1993): Pasado y perspectivas del sistema sindical, Revista de la CEPAL, No 49, LC/G.1757-P, Santiago de Chile, abril.

Calderón G., F., M. Hopenhayn y E. Ottone (1994): Una perspectiva cultural de las propuestas de la CEPAL, Revista de la CEPAL, $\mathrm{N}^{\circ}$ 52, LC/G.1824-P, Santiago de Chile, abril.

Cardoso, F.H. (1977): La originalidad de la copia: la cEPAL y la idea de desarrollo, Revista de la CEPAL, $\mathrm{N}^{\circ}$ 4, Santiago de Chile, segundo semestre.

(2004): Más allá de la economía: interacciones de la política y desarrollo económico, Revista de la CEPAL, No 83, LC/G.2231-P, Santiago de Chile, agosto.

Carlson, B.A. (2002): Educación y mercado del trabajo en América Latina, Revista de la CEPAL, No 77, LC/G.2180-P, agosto.

CEPAL (Comisión Económica para América Latina y el Caribe) (1949): Estudio económico de América Latina 1948, E/CN/.12/82, Nueva York, Naciones Unidas.

(1951): Estudio económico de América Latina 1949, E/CN.12/164/Rev.1, Nueva York, Naciones Unidas.

(1974): Boletín económico de América Latina, vol. 19, $\mathrm{N}^{\circ}$ 1-2, Santiago de Chile.

(2008a): Estudio económico de América Latina y el Caribe, 2007-2008. Política macroeconómica y volatilidad, Santiago de Chile, LC/G.2386-P, Santiago de Chile, octubre. Publicación de las Naciones Unidas, $\mathrm{N}^{\mathbf{o}}$ de venta: S.08.II.G.2.

(2008b): Juventud y cohesión social en Iberoamérica: un modelo para armar, LC/G.2391, Santiago de Chile, octubre,

CEPAL/UNESCO (Comisión Económica para América Latina y el Caribe/ Organización de las Naciones Unidas para la Educación, la
Ciencia y la Cultura) (1992): Educación y conocimiento: eje de la transformación productiva con equidad, Libros de la CEPAL, No 33 (LC/G.1702/Rev.2-P), Santiago de Chile. Publicación de las Naciones Unidas, $\mathrm{N}^{\circ}$ de venta: S.92.II.G.6.

Cohen, E. y R. Franco (1992): Racionalizando la política social: evaluación y viabilidad, Revista de la CEPAL, No 47, LC/G.1739-P, Santiago de Chile, agosto.

Cortés Conde, R. (2001): Raúl Prebisch: los años de gobierno, Revista de la CEPAL, No 75, LC/G.2150-P, Santiago de Chile, diciembre.

Deluchey, J.F. (2005): Architectures de la sécurité intérieure en Amérique latine: entre héritages et nouvelle donne, Revista de la CEPAL, número especial en francés, LC/G.2263-P, Santiago de Chile, junio.

Devlin, R. (1979): Los bancos comerciales y el desarrollo de la periferia: congruencia y conflicto, Revista de la CEPAL, $\mathrm{N}^{\circ} 9$, E/CEPAL/G.1096, Santiago de Chile, diciembre.

(1985): Deuda externa y crisis: el ocaso de la gestión ortodoxa, Revista de la CEPAL, No 27, LC/G.1368-P, Santiago de Chile, diciembre.

(1989): Disyuntivas frente a la deuda externa, Revista de la CEPAL, N $\mathrm{N}^{\circ}$ 37, LC/G.1547-P, Santiago de Chile, abril.

Di Filippo, A. (1998): La visión centro-periferia hoy, Revista de la CEPAL, número extraordinario, LC/G.2037-P, Santiago de Chile, octubre.

Dirven, M. (1993): Integración y desintegración social rural, Revista de la CEPAL, No 51, LC/G.1792-P, Santiago de Chile, diciembre. (2004): El empleo rural no agrícola y la diversidad rural en América Latina, Revista de la CEPAL, No 83, LC/G.2231-P, Santiago de Chile, agosto.

Dourojeanni, A. (1994): La gestión del agua y las cuencas en América Latina, Revista de la CEPAL, № 53, LC/G.1832-P, Santiago de Chile, agosto.

Dosman, E. (2001): Los mercados y el Estado en la evolución del «manifiesto» de Prebisch, Revista de la CEPAL, $\mathrm{N}^{\circ} 75$, LC/G.2150-P, Santiago de Chile, diciembre.

Durán de la Fuente, H. (1991): Contaminación industrial y urbana: opciones de política, Revista de la CEPAL, No 44, LC/G.1667-P, Santiago de Chile, agosto.

Durston, J. (1988): Política social rural en una estrategia de desarrollo sostenido, Revista de la CEPAL, N ${ }^{\circ} 36$, LC/G.1537-P, Santiago de Chile, diciembre.

(1992): Tesis errada sobre la juventud de los años noventa, Revista de la CEPAL, $\mathrm{N}^{\circ} 46, \mathrm{LC} / \mathrm{G} .1717-\mathrm{P}$, Santiago de Chile, abril.

(1993): Los pueblos indígenas y la modernidad, Revista de la CEPAL, N ${ }^{\circ}$ 51, LC/G.1792-P, Santiago de Chile, diciembre.

Escaith, H. (2001): Las economías pequeñas de América Latina y el Caribe, Revista de la CEPAL, N ${ }^{\circ} 74$, LC/G.2135-P, Santiago de Chile, agosto.

Eyzaguirre, N. (1989): El ahorro y la inversión bajo restricción externa y fiscal, Revista de la CEPAL, No 38, LC/G.1570-P, Santiago de Chile, agosto.

Fajnzylber, F. (1981): Reflexiones sobre la industrialización exportadora del Sudeste Asiático, Revista de la CEPAL, No 15, E/CEPAL/G.1187, Santiago de Chile, diciembre.

(1983): La industrialización trunca de América Latina, México, D.F., Editorial Nueva Imagen.

(1988): Competitividad internacional: evolución y lecciones, Revista de la CEPAL, No 36, LC/G.1537-P, Santiago de Chile, diciembre.

(1990): Industrialización en América Latina: de la 'caja negra' al 'casillero vacío': comparación de patrones contemporáneos de industrialización, Cuadernos de la CEPAL, No 60 , LC/G.1534/ Rev.1-P, Santiago de Chile. Publicación de las Naciones Unidas, $\mathrm{N}^{\mathrm{o}}$ de venta: S.89.II.G.5. 
(1991): Inserción internacional e innovación institucional, Revista de la CEPAL, No 44, LC/G.1667-P, Santiago de Chile, agosto.

Faletto, E. (1988): Cultura política y conciencia democrática, Revista de la CEPAL, $\mathrm{N}^{\circ} 35$, LC/G.1527-P, Santiago de Chile, agosto.

(1989): La especificidad del Estado en América Latina, Revista de la CEPAL, No 38, LC/G.1570-P, Santiago de Chile, agosto.

Feres, J.C. y A. León (1990): Magnitud de la situación de la pobreza, Revista de la CEPAL, $\mathrm{N}^{\circ}$ 41, LC/G.1631-P, Santiago de Chile, agosto.

Ferrer, A. (1998): América Latina y la globalización, Revista de la CEPAL, número extraordinario, LC/G.2037-P, Santiago de Chile, octubre.

Ffrench-Davis, R. (1988): Esbozo de un planteamiento neoestructuralista, Revista de la CEPAL, No 34, LC/G.1521-P, Santiago de Chile, abril.

Franco, C. (1989): Participación y concertación en las políticas sociales, Revista de la CEPAL, № 37, LC/G.1547-P, Santiago de Chile, abril.

Frenkel, R. (2003): Globalización y crisis financieras en América Latina, Revista de la CEPAL, No 80, LC/G.2204-P, Santiago de Chile, agosto.

Fuentes, J.A. (1994): El regionalismo abierto y la integración económica, Revista de la CEPAL, N 53, LC/G.1832-P, Santiago de Chile, agosto.

Furtado, C. (1978): Acumulación y creatividad, Revista de la CEPAL, $\mathrm{N}^{\circ}$ 6, Santiago de Chile, segundo semestre.

Gligo, N. (1990): Las cuentas del patrimonio natural y el desarrollo sustentable, Revista de la CEPAL, № 41, LC/G.1631-P, Santiago de Chile, agosto.

(1995): Situación y perspectivas ambientales en América Latina y el Caribe, Revista de la CEPAL, No 55, LC/G.1858-P, Santiago de Chile, abril.

González, N. (1988): Una política económica para el desarrollo, Revista de la CEPAL, $\mathrm{N}^{\circ} 34$, LC/G.1521-P, Santiago de Chile, abril.

(2001): Las ideas motrices de tres procesos de industrialización, Revista de la CEPAL, $\mathrm{N}^{\circ} 75$, LC/G.2150-P, Santiago de Chile, diciembre.

Guerguil, M. (1988): Algunos alcances sobre la definición del sector

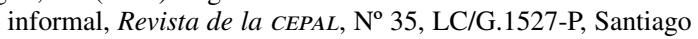
de Chile, agosto.

Graciarena, J. (1976): Poder y estilos de desarrollo: una perspectiva heterodoxa, Revista de la CEPAL, $\mathrm{N}^{\circ}$ 1, Santiago de Chile, primer semestre.

(1988): Una esperanzada visión de la democracia, Revista de la CEPAL, $\mathrm{N}^{\circ} 35$, LC/G.1527-P, Santiago de Chile, agosto.

Gurrieri, A. (2001): Las ideas del joven Prebisch, Revista de la CEPAL, $\mathrm{N}^{\mathrm{o}} 75, \mathrm{LC} / \mathrm{G} .2150-\mathrm{P}$, Santiago de Chile, diciembre.

Halperin, T. (2008): La CEPAL en su contexto histórico, Revista de la CEPAL, N ${ }^{\circ}$ 94, LC/G.2357-P, Santiago de Chile, abril.

Harker, T. (1987): La política del sector agrícola y la planificación macroeconómica, Revista de la CEPAL, No 33, LC/G.1491-P, Santiago de Chile, diciembre.

Heymann, D. (2000): Grandes perturbaciones macroeconómicas, expectativas y respuestas de política, Revista de la CEPAL, $\mathrm{N}^{\circ} 70$, LC/G.2095-P, Santiago de Chile, abril.

Hofman, A. A. y R. Buitelaar (1994): Ventajas comparativas extraordinarias y crecimiento el caso a largo plazo: el caso de Ecuador, Revista de la CEPAL, No 54, LC/G.1845-P, Santiago de Chile, diciembre

Hopenhayn, M. (1992): ¿Pensar lo social sin planificación ni revolución?, Revista de la CEPAL, N 48, LC/G.1748-P, Santiago de Chile, diciembre.

(2001): Viejas y nuevas formas de la ciudadanía, Revista de la CEPAL, $\mathrm{N}^{\circ} 73$, LC/G.2130-P, Santiago de Chile, abril.

Ibarra, D. (2004): Los laberintos del orden internacional: la importación de reformas, Revista de la CEPAL, $\mathrm{N}^{\circ}$ 82, LC/G.2220-P, Santiago de Chile, abril.
Iglesias, E.V. (1983): Reflexiones sobre la economía latinoamericana durante 1982, Revista de la CEPAL, N 19, E/CEPAL/G.1229, Santiago de Chile, abril.

(2006): El papel del Estado y los paradigmas económicos en América Latina, Revista de la CEPAL, N 90, LC/G.2323-P, Santiago de Chile, diciembre.

Infante B., R. y E. Klein (1991): Mercado latinoamericano del trabajo en 1950-1990, Revista de la CEPAL, $\mathrm{N}^{\circ} 45, \mathrm{LC} / \mathrm{G} .1687-\mathrm{P}$, Santiago de Chile, diciembre.

Jiménez, J.P. y V. Tromben (2006): Política fiscal y bonanza: impacto del aumento de los precios de los productos no renovables en América Latina y el Caribe, Revista de la CEPAL, $\mathrm{N}^{\circ} 90$, LC/G.2323-P, Santiago de Chile, diciembre.

Jiménez, L.F. y J. Cuadros (2003): Ampliación de la cobertura de los sistemas de pensiones en América Latina, Revista de la CEPAL, $\mathrm{N}^{\circ}$ 79, LC/G.2200-P, Santiago de Chile, abril.

Jovel, J.R. (1989): Los desastres naturales y su incidencia económicosocial, Revista de la CEPAL, N 38 , LC/G.1570-P, Santiago de Chile, agosto.

Katz, J. (1998): Aprendizaje tecnológico ayer y hoy, Revista de la CEPAL, número extraordinario, LC/G.2037-P, Santiago de Chile, octubre.

(2000): Cambios estructurales y productividad en la industria latinoamericana, 1970-1996, Revista de la CEPAL, $\mathrm{N}^{\mathrm{o}} 71$, LC/G.2060-P, Santiago de Chile, agosto.

Kervran, D.D. (2005): Les politiques de conservation de la nature en Amérique latine: au coeur de l'internationalisation et de la convergence des ordres politiques, Revista de la CEPAL, número especial en francés, LC/G.2243-P, Santiago de Chile, junio.

Klein, E. y V.E. Tokman (2000): La estratificación social bajo tensión en la era de la globalización, Revista de la CEPAL, $\mathrm{N}^{\circ} 72$, LC/G.2120-P, Santiago de Chile, diciembre.

Krawczyk, M. (1990): La creciente presencia de la mujer en el desarrollo, Revista de la CEPAL, N 40, LC/G.1613-P, Santiago de Chile, abril.

(1993): Mujeres en la región: los grandes cambios, Revista de la CEPAL, Nº 49, LC/G.1757-P, Santiago de Chile, abril.

Kuwayama, M. (1989): El potencial tecnológico del sector primario exportador, Revista de la CEPAL, № 39, LC/G.1583-P, Santiago de Chile, diciembre.

Lahera, E. (1988): Cambio técnico y reestructuración productiva, Revista de la CEPAL, $\mathrm{N}^{\circ}$ 36, LC/G.1537-P, Santiago de Chile, diciembre.

(1990): El Estado y la transformación productiva con equidad, Revista de la CEPAL, No 42, LC/G.1642-P, Santiago de Chile, diciembre.

López, M. C. y E. M. Pollack (1989): La incorporación de la mujer en las políticas de desarrollo, Revista de la CEPAL, $\mathrm{N}^{\mathrm{o}} 39$, LC/G.1583-P, Santiago de Chile, diciembre.

López Cordovez, L. (1987): Crisis, políticas de ajuste y agricultura, Revista de la CEPAL, № 33, LC/G.1491-P, Santiago de Chile, diciembre.

Machinea, J.L. y D. Titelman (2007): ¿Un crecimiento menos volátil? El papel de las instituciones financieras regionales, Revista de la CEPAL, N 91, LC/G.2333-P, Santiago de Chile, abril.

Martner Fanta, R. (2000): Los estabilizadores fiscales automáticos, Revista de la CEPAL, N 70, LC/G.2095-P, Santiago de Chile, abril.

Massad, C. (1983): El costo real de la deuda externa para el acreedor y para el deudor, Revista de la CEPAL, $\mathrm{N}^{\circ} 19, \mathrm{E} / \mathrm{CEPAL} / \mathrm{G} .1229$, Santiago de Chile, abril.

Medina Echavarría, J. (1976): América Latina en los escenarios posibles de la distensión, Revista de la CEPAL, $\mathrm{N}^{\circ} 2$, Santiago de Chile, segundo semestre.

(1977): Apuntes acerca del futuro de las democracias occidentales, Revista de la CEPAL, $\mathrm{N}^{\circ} 4$, Santiago de Chile, segundo semestre. 
Meller, P. (1989): En torno a la doble condicionalidad del FMI y del Banco Mundial, Revista de la CEPAL, No 37, LC/G.1547-P, Santiago de Chile, abril.

Mesa-Lago, C. (1996): Las reformas de las pensiones en América Latina y la posición de los organismos internacionales, Revista de la CEPAL, $\mathrm{N}^{\circ}$ 60, LC/G.1943-P, Santiago de Chile, diciembre.

(2004): Evaluación de un cuarto de siglo de reformas estructurales de pensiones en América Latina, Revista de la CEPAL, No 84, LC/G.2258-P, Santiago de Chile, diciembre.

Moguillansky, G. (2002): Inversión y volatilidad financiera en América Latina, Revista de la CEPAL, No 77, LC/G.2180-P, Santiago de Chile, agosto.

Morley, S.A. (2000): Efectos del crecimiento y las reformas económicas sobre la distribución del ingreso en América Latina, Revista de la CEPAL, $\mathrm{N}^{\circ} 71, \mathrm{LC} / \mathrm{G} .2060-\mathrm{P}$, Santiago de Chile, agosto.

Mortimore, M. (1989): Conductas de los bancos acreedores de América Latina, Revista de la CEPAL, No 37, LC/G.1547-P, Santiago de Chile, abril.

Ocampo, J.A. (1999): La reforma financiera internacional: una agenda ampliada, Revista de la CEPAL, $\mathrm{N}^{\circ}$ 69, LC/G.2067-P, Santiago de Chile, diciembre.

(2001): Raúl Prebisch y la agenda del desarrollo en los albores del siglo XXI, Revista de la CEPAL, N ${ }^{\circ} 75$, LC/G.2150-P, Santiago de Chile, diciembre.

(2007): La macroeconomía de la bonanza económica latinoamericana, Revista de la CEPAL, LC/G.2347-P, Santiago de Chile, diciembre.

Ocampo, J.A. y M.A. Parra (2003): Los términos de intercambio de los productos básicos en el siglo XX, Revista de la CEPAL, $\mathrm{N}^{\circ}$ 79, LC/G.2200-P, Santiago de Chile, abril.

O'Connell, A. (2001): El regreso de la vulnerabilidad y las ideas tempranas de Prebisch sobre el "ciclo argentino", Revista de la CEPAL, $\mathrm{N}^{\circ} 75$, LC/G.2150-P, Santiago de Chile, diciembre.

Ortega, E. (1988): La agricultura en la óptica de la CEPAL, Revista de la CEPAL, $\mathrm{N}^{\mathrm{o}} 35, \mathrm{LC} / \mathrm{G} .1527-\mathrm{P}$, Santiago de Chile, agosto.

Paunovic, I. (2005): Sostenibilidad de la deuda pública en los países norteños de América Latina, Revista de la CEPAL, $\mathrm{N}^{\circ} 87$, LC/G.2287-P, Santiago de Chile, diciembre.

Peres Núñez, W. (1993): Internacionalización de empresas industriales latinoamericanas, Revista de la CEPAL, No 49, LC/G.1757-P, Santiago de Chile, abril.

(1994): Política de competitividad, Revista de la CEPAL, $\mathrm{N}^{\mathrm{0}}$ 53, LC/G.1832-P, Santiago de Chile, agosto.

Pérez, C. (2001): Cambio tecnológico y oportunidades de desarrollo como blanco móvil, Revista de la CEPAL, N ${ }^{\circ} 75$, LC/G.2150-P, Santiago de Chile, diciembre.

Pinto Santa Cruz, A. (1970): Naturaleza e implicaciones de la 'heterogeneidad estructural' de la América Latina, El trimestre económico, vol. 37, $\mathrm{N}^{\circ}$ 145, México, D.F., Fondo de Cultura Económica, enero-marzo.

(1976): Notas sobre los estilos de desarrollo en América Latina, Revista de la CEPAL, $\mathrm{N}^{\circ} 1$, Santiago de Chile, primer semestre.

Pollock, D., D. Kerner y J.L. Love (2001): Entrevista inédita a Prebisch: logros y deficiencias de la CEPAL, Revista de la CEPAL, $\mathrm{N}^{\circ} 75$, LC/G.2150-P, Santiago de Chile, diciembre.

Prebisch, R. (1976): Crítica al capitalismo periférico, Revista de la CEPAL, $\mathrm{N}^{\circ}$ 1, Santiago de Chile, primer semestre.

(1978): Estructura socioeconómica y crisis del sistema: reflexiones al cumplirse nuestros primeros treinta años, Revista de la CEPAL, $\mathrm{N}^{\circ}$ 6, Santiago de Chile, segundo semestre.

(1979): Las teorías neoclásicas del liberalismo económico, Revista de la CEPAL, $\mathrm{N}^{\circ}$ 7, E/CEPAL/1084, Santiago de Chile, abril.

(1980): Hacia una teoría de la transformación, Revista de la CEPAL, $\mathrm{N}^{\circ} 10$, Santiago de Chile, abril.

(1981): Capitalismo periférico. Crisis y transformación, México, D.F., Fondo de Cultura Económica.
(1983): Cinco etapas de mi pensamiento sobre el desarrollo, El trimestre económico, vol. 50(2), № 198, México, D.F., Fondo de Cultura Económica, abril-junio.

Ramos, J. (1989): La macroeconomía neokeynesiana vista desde el Sur, Revista de la CEPAL, No 38, LC/G.1570-P, Santiago de Chile, agosto.

Ricúpero, R. (2004): La renovada contemporaneidad de Raúl Prebisch, Revista de la CEPAL, N 84, LC/G.2258-P, Santiago de Chile, diciembre.

Rodrik, D. (2005): Políticas de diversificación económica, Revista de la CEPAL, $\mathrm{N}^{\circ} 87$, LC/G.2287-P, Santiago de Chile, diciembre.

Rodríguez Vignoli, J. (2005): Reproducción en la adolescencia: el caso de Chile y sus implicaciones de política, Revista de la CEPAL, $\mathrm{N}^{\circ}$ 86, LC/G.2282-P, Santiago de Chile, agosto.

Rodríguez, O. (2001): Prebisch: actualidad de sus ideas básicas, Revista de la CEPAL, No 75, LC/G.2150-P, Santiago de Chile, diciembre.

(2006): El estructuralismo latinoamericano, México, D.F., CEPAL-Siglo XXI Editores, noviembre.

Rodríguez Noboa, P. (1991): La selectividad como eje de las políticas sociales, Revista de la CEPAL, No 44, LC/G.1667-P, Santiago de Chile, agosto.

Rosales V. O. (1994): Política industrial y fomento de la competitividad, Revista de la CEPAL, No 53, LC/G.1832-P, Santiago de Chile, agosto.

Rosenbluth, G. (1994): Informalidad y pobreza en América Latina, Revista de la CEPAL, $\mathrm{N}^{\circ}$ 52, LC/G.1824-P, Santiago de Chile, abril.

Rosenthal, G. (1988): La CEPAL en su cuadragésimo aniversario: continuidad y cambio, Revista de la CEPAL, N 35 , LC/G.1527-P, Santiago de Chile, agosto.

(1993): La integración regional en los años noventa, Revista de la CEPAL, No 50, LC/G.1767-P, Santiago de Chile, agosto.

Sánchez Albavera, F. (1993): El actual debate sobre los recursos naturales, Revista de la CEPAL, N 51, LC/G.1792-P, Santiago de Chile, diciembre.

(1995): Globalización y reestructuración energética en América Latina, Revista de la CEPAL, No 56 , LC/G.1874-P, Santiago de Chile, agosto.

Saraví, G.A. (2004): Segregación urbana y espacio público: los jóvenes en enclaves de pobreza estructural, Revista de la CEPAL, $\mathrm{N}^{\circ} 83$, LC/G.2231-P, Santiago de Chile, agosto.

Schejtman, A. (1988): La seguridad alimentaria: tendencias e impacto de la crisis, Revista de la CEPAL, No 36, LC/G.1537-P, Santiago de Chile, diciembre.

Schkolnik, S. y J. Chackiel (2004): Los sectores rezagados en la transición de la fecundidad en América Latina, Revista de la CEPAL, $\mathrm{N}^{\circ}$ 83, LC/G.2231-P, Santiago de Chile, agosto.

Sojo, A. (1990): Naturaleza y selectividad de la política social, Revista de la CEPAL, $\mathrm{N}^{\circ}$ 41, LC/G.1631-P, Santiago de Chile, agosto.

(2001): Reformas de gestión en salud en América Latina, Revista de la CEPAL, No 74, LC/G.2135-P, Santiago de Chile, agosto.

Stallings, B. y J. Weller (2001): El empleo en América Latina, base fundamental de la política social, Revista de la CEPAL, $\mathrm{N}^{\mathrm{o}} 75$, LC/G.2150-P, Santiago de Chile, diciembre.

Stiglitz, J.E. (2003): El rumbo de las reformas. Hacia una nueva agenda para América Latina, Revista de la CEPAL, No 80, LC/G.2204-P, Santiago de Chile, agosto.

Sunkel, O. (1980): La interacción entre los estilos de desarrollo y el medio ambiente en América Latina, Revista de la CEPAL, $\mathrm{N}^{\mathrm{o}} 12$, E/CEPAL/G.1130, Santiago de Chile, diciembre.

(1989): Institucionalismo y estructuralismo, Revista de la CEPAL, N ${ }^{\circ} 38$, LC/G.1570-P, Santiago de Chile, agosto.

(1998): Desarrollo e integración regional: ¿otra oportunidad para una promesa incumplida?, Revista de la CEPAL, número extraordinario, LC/G.2037-P, Santiago de Chile, octubre. 
Sunkel, O. y G. Zuleta (1990): Neoestructuralismo versus neoliberalismo en los años noventa, Revista de la CEPAL, No 42, LC/G.1642-P, Santiago de Chile, diciembre.

Tedesco, J.C. y N. López (2002): Desafíos a la educación secundaria en América Latina, Revista de la CEPAL, No 76, LC/G.2175-P, Santiago de Chile, abril.

Tokman, V.E. (1988): Empleo urbano: investigación y políticas en América Latina, Revista de la CEPAL, No 34, LC/G.1521-P, Santiago de Chile, abril.

Titelman Kardonsky, D. (1999): Reformas al financiamiento del sistema de salud en Chile, Revista de la CEPAL, N 69, LC/G.2067-P, Santiago de Chile, diciembre.

Titelman Kardonsky, D. y A. Uthoff (2003): El papel del aseguramiento en la protección social, Revista de la CEPAL, N ${ }^{\circ}$ 81, LC/G.2216-P, Santiago de Chile.

Tomic, T. (1992): Participación y medio ambiente, Revista de la CEPAL, $\mathrm{N}^{\circ}$ 48, LC/G.1748-P, Santiago de Chile, diciembre.

Torres Olivos, M. (comp.) (2006): Fernando Fajnzylber, Una visión renovadora del desarrollo en América Latina, Libros de la CEPAL, Nº 92, LC/G.2322-P, Santiago de Chile, Comisión Económica para América Latina y el Caribe (CEPAL), noviembre.

Urquidi, V.L. (1998): Incidentes de integración en Centroamérica y Panamá, 1952-1958, Revista de la CEPAL, número extraordinario, LC/G.2037-P, Santiago de Chile, octubre.

Uthoff, A. (1995): Reformas a los sistemas de pensiones en América Latina y el Caribe, Revista de la CEPAL, N ${ }^{\circ}$ 56, LC/G.1874-P, Santiago de Chile, agosto.
(2002): Mercados de trabajo y sistemas de pensiones, Revista de la CEPAL No 78, LC/G.2187-P, Santiago de Chile, diciembre.

(2006): Brechas del Estado de bienestar y reformas a los sistemas de pensiones en América Latina, Revista de la CEPAL, $N^{\circ} 89$, LC/G.2312-P, Santiago de Chile.

Valenzuela, R. (1991): El que contamina, paga, Revista de la CEPAL, $\mathrm{N}^{\circ} 45$, LC/G.1687-P, Santiago de Chile, diciembre.

Vergara, S. (2005): Dinámica laboral de la industria en Chile, Revista de la CEPAL, $\mathrm{N}^{\circ}$ 86, LC/G.2282-P, Santiago de Chile, agosto.

Villatoro S. P. (2005): Programas de transferencias monetarias condicionadas: experiencias en América Latina, Revista de la CEPAL, $\mathrm{N}^{\mathrm{o}}$ 86, LC/G.2282-P, Santiago de Chile, agosto.

Weller, J. (2007): La inserción laboral de los jóvenes: características, tensiones y desafíos, Revista de la CEPAL, N ${ }^{\circ}$ 92, LC/G.2339-P, Santiago de Chile, agosto.

Willmore, L. (1989): La promoción de exportaciones y la sustitución de importaciones en la industria centroamericana, Revista de la CEPAL, No 38, LC/G.1570-P, Santiago de Chile, agosto.

Wolfe, M. (1976): Enfoques del desarrollo: ¿de quién y hacia qué?, Revista de la CEPAL, $\mathrm{N}^{\circ}$ 1, Santiago de Chile, primer semestre.

(1990): Las estructuras sociales y la democracia en los años noventa, Revista de la CEPAL, $\mathrm{N}^{\circ}$ 40, LC/G.1613-P, Santiago de Chile, abril.

(1991): Perspectivas sobre la equidad, Revista de la CEPAL, $\mathrm{N}^{\circ}$ 44, LC/G.1667-P, Santiago de Chile, agosto. 